\title{
Proximity Constraints in Deformable Models for Cortical Surface Identification
}

\author{
David MacDonald, David Avis, and Alan C. Evans \\ McConnell Brain Imaging Centre, Montréal Neurological Institute, \\ McGill University, Montréal, Canada \\ davidQbic.mni.megill.ca \\ http://www.bic.mni.mcgill.ca
}

\begin{abstract}
Automatic computer processing of large multi-dimensional images such as those produced by magnetic resonance imaging (MRI) is greatly aided by deformable models. A general method of deforming polyhedra is presented here, with two novel features. Firstly, explicit prevention of non-simple (self-intersecting) surface geometries is provided, unlike conventional deformable models which merely discourage such behaviour. Secondly, simultaneous deformation of multiple surfaces with inter-surface proximity constraints provides a greater facility for incorporating model-based constraints into the process of image recognition. These two features are used advantageously to automatically identify the total surface of the cerebral cortical gray matter from normal human MR images, accurately locating the depths of the sulci even where under-sampling in the image obscures the visibility of sulci. A large number of individual surfaces $(\mathrm{N}=151)$ are created and a spatial map of the mean and standard deviation of the cerebral cortex and the thickness of cortical gray matter are generated. Ideas for further work are outlined.
\end{abstract}

\author{
Contact: David MacDonald \\ Address: McConnell Brain Imaging Centre, Webster 2B, \\ Montréal Neurological Institute, \\ 3801 University, Montréal, Québec, H3A 2B4 \\ Fax: $\quad$ 514-398-8948
}

Keywords: Image Segmentation, Surface Deformation, Cerebral Cortex

\section{Introduction}

Digitally acquired information is increasingly being used in medical fields for surgery planning and intra-operative navigation, diagnosis and monitoring of disease, as well as in investigations of normal and pathological anatomy. In the realm of computational neuroanatomy, large amounts of three- and fourdimensional anatomical and functional information are available in the form of such modalities as magnetic resonance imaging (MRI), positron emission tomography (PET), and computed tomography (CT). The diverse nature of the image 
data, in addition to the large size of individual images, motivates researchers to explore automated methods of processing the data into forms more useful for subsequent analysis. Deformable models provide a promising technique for registration, segmentation, and matching tasks in computational neuroanatomy, by combining the bottom-up approach of edge detection with the top-down approach of model-based geometric constraints. The active contour method of Kass et al. [1], commonly referred to as "SNAKES", has been the foundation upon which deformation methods have been based. Essentially, a two or three dimensional spline is assigned an energy function which consists of a stretching and smoothing term based on first and second derivatives, and an image term which decreases in energy as the spline moves closer to image boundaries. Numerical integration techniques deform the spline from a starting position to a minimum energy configuration, which represents a compromise between the shape constraints and the image edge features. Many adaptations of deformable models in medical imaging have since been presented [2-6], and a survey of these methods is presented in [7]. Here we address two of the limitations of conventional methods. Firstly, deformable surfaces typically use stretching and bending constraints for a regularization effect which penalizes but does not prevent non-simple (selfintersecting) surface geometries. Secondly, single surface deformation methods are sensitive to partial volume effects in images due to under-sampling. Some existing methods $([8,9])$ attempt to disambiguate the location of cerebral cortical surfaces in human MR images by combining information from both gray and white matter image boundaries. This idea is extended here to present a novel formulation of deformable polyhedra which incorporates a variety of proximity constraints in order to identify the entire cortical surface of the human brain from MR images of normal volunteers. The use of inter- and intra-surface intersection avoidance as well as a two-surface model of the inner and outer boundaries of the cortical gray mantle results in a fully automatic identification of the entire surface, even in deep, narrow sulci which may be confounded by partial volume effects. Surfaces are guaranteed not to intersect themselves or each other. The formulation of this method as a minimization problem is described in the next section. Application to a population of datasets, inter-subject averaging, and mapping of cortical surfaces to simpler parameter spaces is described. Finally, ideas for further application are presented.

\section{Method}

The essence of the method is the formulation of an objective function which when minimized provides a solution to an image recognition problem. The domain of the function is the set of vertex coordinates describing one or more polyhedra to be deformed, and the range is a scalar value representing a goodness of fit of the polyhedra to the target data. The objective function and its terms are described, followed by the method of minimization. 


\subsection{Objective Function}

The objective function, $O(S)$, may be defined generally as a weighted sum of $N_{t}$ terms, each of which may be thought of as a data or model term, depending on whether it constrains the deforming polyhedra to match image data or some model-based a priori information:

$$
O(S)=\sum_{k=1}^{N_{t}} w_{k} T_{k},
$$

where $w_{k}$ is a weighting factor, $S$ is a set of $N_{s}$ deforming polyhedral surfaces,

$$
S=\left\{S_{i}: S_{i} \text { is a polyhedral surface, } 1 \leq i \leq N_{s}\right\},
$$

and $\hat{S}$ is a set of $N_{s}$ model polyhedral surfaces where each $\hat{S}_{i}$ has the same topology as $S_{i}$. Each term, $T_{k}$, is formulated as:

$$
T_{k}(S)=W\left(D_{k}(S)\right),
$$

where $D_{k}(S)$ is a signed scalar measure of deviation from some ideal, and $W(x)$ is a general weighting function. Usually this is just a squaring function, $W(x)=x^{2}$, but the function $W(x)$ can be also be used as an attenuation function by increasing the cost sharply when the measure of deviation passes a certain threshold. Each objective term is described here for the simple case where $W(x)$ is a squaring function. As a prolog, some definitions are presented:

$$
\begin{array}{ll}
\bar{x}_{v}=\left(x_{v}, y_{v}, z_{v}\right), & \text { the 3D position of vertex } v \text { in a deforming polyhedral } \\
& \text { mesh, } \\
\bar{x}_{v}=\left(\hat{x}_{v}, \hat{y}_{v}, \hat{z}_{v}\right), & \text { the 3D position of vertex } v \text { in a static model polyhedral } \\
& \text { mesh, } \\
n_{v}, & \text { the number of vertices in a polyhedral mesh. } \\
n_{e}, & \text { the number of edges in a polyhedral mesh. } \\
n_{p}, & \text { the number of polygons in a polyhedral mesh. and } \\
m_{v}, & \text { the number of neighbours of vertex } v, \\
d(\bar{x}, \bar{y}), & \text { the Euclidean distance between two three dimensional } \\
& \text { points, } \\
n_{v, j}, & \text { the } j \text { 'th neighbour of vertex } v, \text { and } \\
\bar{N}_{v}, & \text { the surface normal at vertex } v, \text { defined as the unit nor- } \\
& \text { mal to the polygon consisting of the counterclockwise } \\
& \text { ordered neighbours of the vertex. }
\end{array}
$$

Image Term The image term is based on the distance from each vertex to the nearest image boundary in the direction of the local surface normal, and is expressed as

$$
T_{\text {boundary_dist }}=\sum_{v=1}^{n_{v}} d_{B}\left(\bar{x}_{v}, \bar{N}_{v}, t\right)^{2}
$$


where $d_{B}\left(\bar{x}_{v}, \bar{N}_{v}, t\right)$ is the distance to the nearest image contour of the threshold, $t$, from the vertex, $v$, along the line defined by the surface normal, $\bar{N}_{v}$. The explicit search in both directions along the surface normal increases the power of locating image boundaries that are relatively far from the current surface position. This term may be modified to use first and second derivative information, or improved by over-sampling between vertices.

Stretching Term The stretch term increases as lengths between vertices are stretched or compressed relative to a user-defined model surface representing the ideal lengths,

$$
T_{\text {stretch }}=\sum_{v=1}^{n_{v}} \sum_{j=1}^{m_{v}}\left(\frac{d\left(\bar{x}_{v}, \bar{x}_{n_{v, j}}-L_{v, j}\right.}{L_{v, j}}\right)^{2},
$$

where $L_{v, j}$, the ideal length of an edge, is defined as the corresponding length in the model polyhedron:

$$
L_{v, j}=d\left(\hat{x}_{v}, \hat{x}_{n_{v, j}}\right) .
$$

The intended effect of this term is to make distances between corresponding pairs of vertices on the model and deformed surface roughly equivalent, and is analogous to the term involving the magnitude of the first derivative of the spline in the original Snakes formulation.

Bending Term The bending term provides a measure of deviation from a model shape based on an estimate of local curvature, and is analogous to the second derivative term in the Snakes formulation,

$$
T_{b e n d}=\sum_{e=1}^{n_{e}}(a(S, e)-a(\hat{S}, e))^{2},
$$

where $a(S, e)$ is the signed angle between the two polygons adjacent to the edge, $e$. This term is intended to be used for shape-based matching and segmentation.

Self-Proximity Term and Inter-Surface Proximity Term The previous three terms are found in some form in most conventional deformable models. Here we introduce the self-proximity term, which measures the proximity of pairs of non-adjacent polygons in a surface,

$$
T_{\text {self-proximity }}=\sum_{i=1}^{n_{p-1}} \sum_{j=i+1}^{n_{p}} \begin{cases}\left(\hat{d}\left(P_{i}, P_{j}\right)-d_{i, j}\right)^{2}, & \text { if } \hat{d}\left(P_{i}, P_{j}\right)<d_{i, j} \\ 0, & \text { otherwise },\end{cases}
$$

where $\hat{d}\left(T_{i}, T_{j}\right)$ is the smallest Euclidean distance between the $i$ 'th polygon, $P_{i}$, and the $j$ 'th polygon, $P_{j}$, and $d_{i, j}$ is a distance threshold. In practice, pairs of adjacent polygons are not included in the above equation, as their $\hat{d}\left(P_{i}, P_{j}\right)$ is a 
constant zero value for any deformation of the polyhedra. The self-proximity term is used to explicitly prevent non-simple topologies by assigning a prohibitively high cost to self-intersecting topologies. The inter-surface proximity term, $T_{\text {surface-surface }}$, is formulated in a similar fashion, and is used to prevent two surfaces from coming within a certain distance of each other.

Vertex-Vertex Proximity Constraints Two surfaces may be designated to prefer to stay a certain distance apart by defining a term constraining the desired distance between specific points on the two surfaces:

$$
T_{\text {vertex-vertex }}=\left(d\left(\bar{x}_{v}, \bar{x}_{w}\right)-d_{B}\right)^{2}
$$

where $d_{B}$ is the preferred distance between vertex $v$ on one surface and vertex $w$ on a second surface. This term keeps specific points of two surfaces a fixed distance apart, but does not explicitly prevent inter-surface intersection, which is achieved by the inter-surface proximity term defined previously.

\subsection{Minimization of Objective Function}

Deformation of polyhedra is achieved by minimization of the objective function using a conjugate gradient method, which involves iteratively computing a derivative direction and a line minimization along a direction computed from successive derivatives. In order to increase the chances of finding the global minimum, a multi-scale approach is employed. Deformation begins with a lowresolution initial guess for each of the polyhedral surfaces being deformed, which may be a hand-crafted model or statistically generated approximation to the surfaces being identified. The low-resolution surfaces are deformed to fit blurred image data, then resampled to contain more triangles. The resampled surfaces are then deformed to fit a less blurred version of the image data, and the process repeated until the desired resolution is achieved. Typically, triangles with lengths of one millimetre are sufficient to capture the surfaces in the MR data being segmented.

\section{Solving Partial Volume Effects with a Double Surface Model}

One of the most interesting applications of this general deformation framework is the identification of deep, narrow sulci that are obscured by partial volume effects. Figure 1a illustrates a cross section through a three dimensional simulated brain phantom. Conventional deformable methods find the sulcus in the white matter (Fig. 1c), but fail to find a sulcus in the gray matter (Fig. 1b) due to partial volume effects in the image. The new deformation method successfully locates a reasonable approximation to the gray matter sulcus (shown in three dimensions in Fig. 1d), using a double surface formulation. The gray-cerebrospinal fluid (CSF) (Fig. 1e) and the gray-white (Fig. 1f) surfaces are simultaneously 
deformed to fit the image, with the constraint that the two surfaces prefer to be a specific distance apart. While neuroanatomical estimates of gray matter thickness vary from three to seven or more millimetres, it was felt that for a preliminary evaluation of this method, five millimetres was a reasonable constraint on the thickness, with a range of plus or minus two millimetres. The gray-CSF surface follows gray-white surface deep into the sulci, and self-proximity constraints prevent it from intersecting itself as the two boundaries of the sulcus are pushed together.

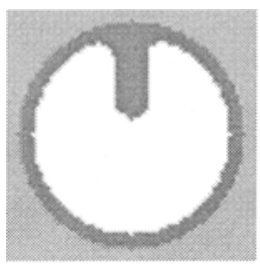

a)

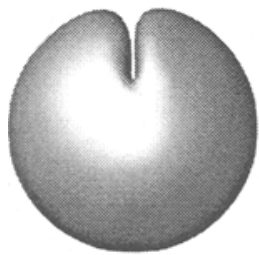

d)

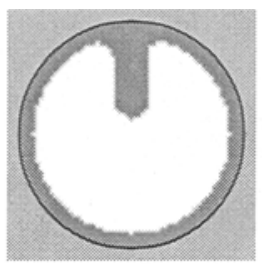

b)

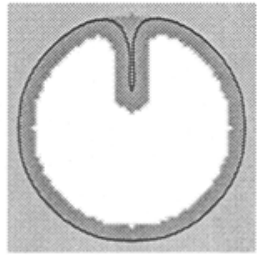

e)

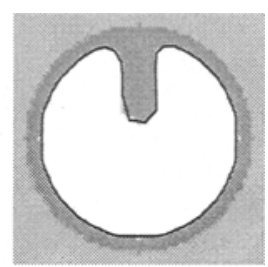

c)

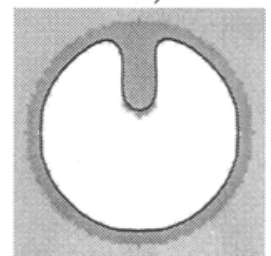

f)

Fig. 1. a) Cross section of image representing sulcus obscured by partial volume. b) Cross section of apparent gray-CSF boundary. c) Cross section of apparent gray-white boundary. d) Results of dual-model deformation. e) Dual-model gray-CSF surface. f) Dual-model gray-CSF surface.

\section{Application to Cortical Surface Segmentation}

The deformation method presented is applied to a large number of normal human MR images to automatically identify the total surface of the cerebral cortical gray matter. T1-weighted, T2-weighted, and PD-weighted images are acquired at a isotropic sampling of one millimetre. The images are corrected for RF inhomogeneity artifacts [10], linearly transformed into a stereotaxic coordinate system [11], and classified into gray matter, white matter, and CSF. The identification of the cortical surface is accomplished with two steps. The first step is use high stretching and bending weights to rigidly deform a coarse cortex mask to fit the classified volume, to remove non-cerebral white matter from the volume. Then the dual-surface deformation described previously is performed on the masked volume, in a multi-scale fashion. The initial surfaces contain 320 triangles, and after several iterations of deformation and sub-sampling the surfaces, the resulting surfaces contain about 90000 triangles. The segmentation for a single 
subject takes about 100 hours of time on a Silicon Graphics Origin 200 R10000 processor running at 180 megahertz. Figure 2 shows the right view of the outer surface of a cerebral cortex, as well as cross sections of the surface superimposed on the classified volume.

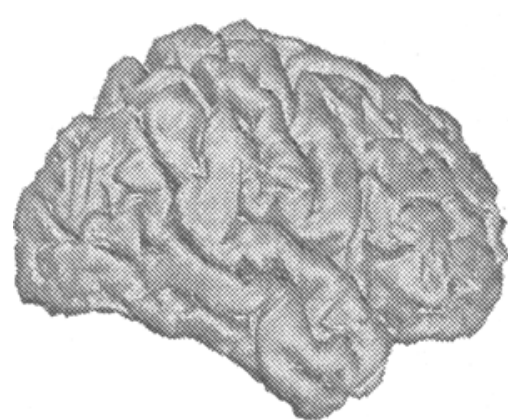

a)

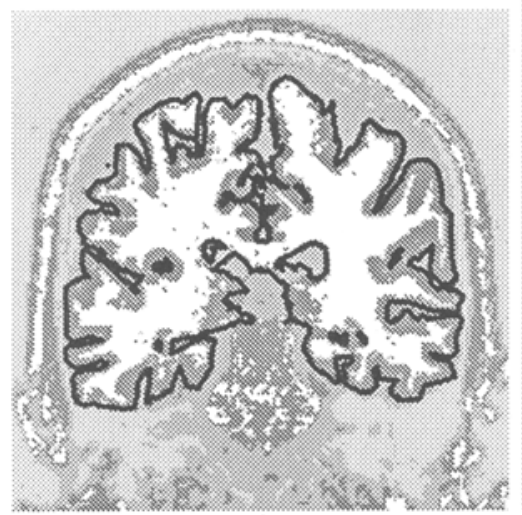

c)

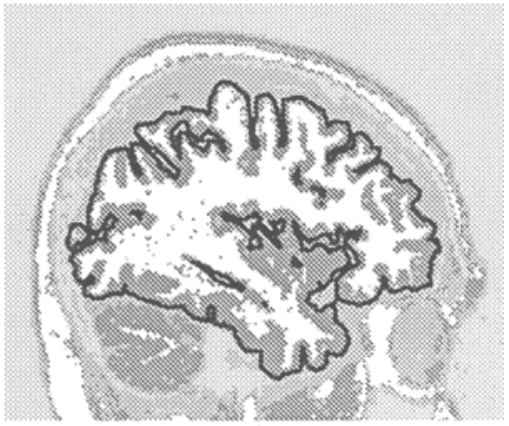

b)

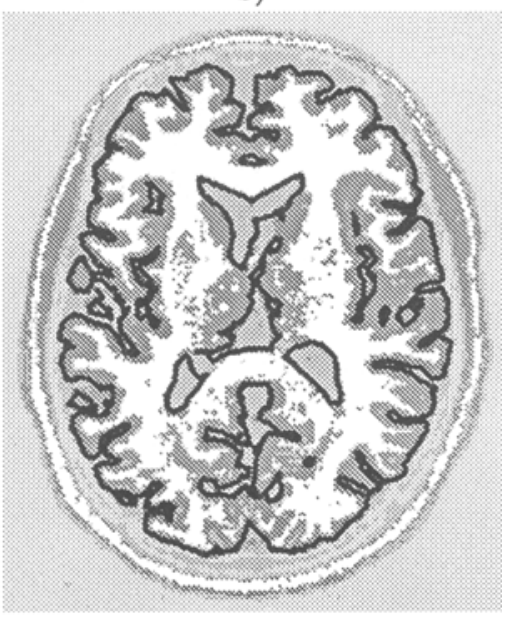

d)

Fig. 2. a) Gray-CSF boundary automatically extracted. b) Sagittal section. c) Coronal section. d) Transverse section.

Choice of Weights A drawback of most deformable methods is the necessity to balance the various objective terms by carefully choosing weights. Most deformable methods use some sort of constraint on stretching and/or bending as regularization terms to avoid degenerate deformation configurations. In the experiments with the current formulation, it has been found that the self-proximity term provides an alternative to the conventional regularization by preventing self intersections, and therefore, it is possible that the stretching and bending terms may be discarded when shape constraints are not needed. This reduces the num- 
ber of arbitrary weights that must be chosen, and a method of assigning the remaining weights has been devises. The image term is arbitrarily assigned a value of one, and the weighting functions for the surface proximity terms are constructed in such a way that geometric measures are specified, rather than weights. For instance, the self-proximity term is constructed so that above 0.25 millimetres the term is 0 , and below .25 millimetres, the weight increases from $10^{-10}$ at 0.25 millimetres to $10^{10}$ at .01 millimetres. This large range of weights works for almost any application, and only the millimetre thresholds for proximity constraints must be chosen.

\subsection{Averaging and Mapping to Two Dimensions}

The surfaces deformed have a topology based on a triangulation of a sphere, and there exists a one-to-one mapping between points on any two deformed surfaces or between a deformed surface and a sphere. The result is that the convoluted cortical surface can be mapped onto a sphere, and sets of deformed surfaces may be averaged into a mean surface. Figure 3a shows the average of 151 individual cerebral cortical surfaces automatically identified by the deformation method. The average curvature of the surfaces is mapped onto the average surface where black areas correspond to sulci, and brighter areas are gyri. Figure $3 \mathrm{~b}$ shows the same curvature information mapped onto a unit sphere. Aside from the visual simplification aspect, it is possible that some types of analysis may be more easily performed in the two dimensional parameter space of the sphere or on the average surface. Depending on the specific analysis requirements, it may be necessary to perform a further step of warping within the two dimensional space, for instance, in order to make the mapping preserve distances, angles, or areas.

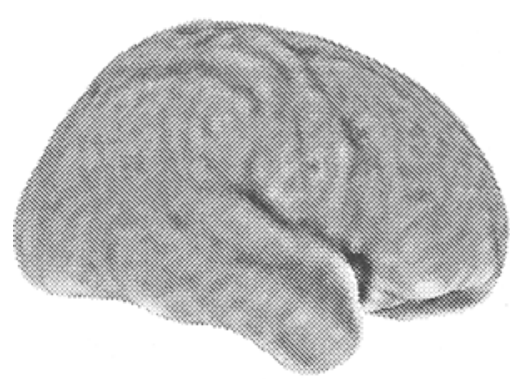

a)

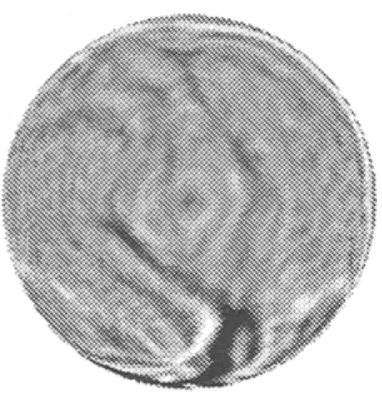

b)

Fig. 3. Curvature of cortex from Fig. 2a mapped onto a) the average surface, and b) a sphere. 


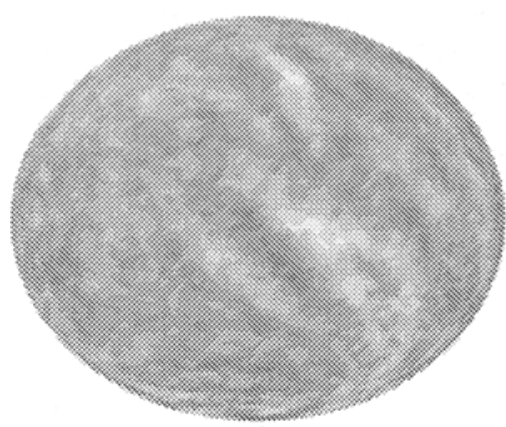

a)

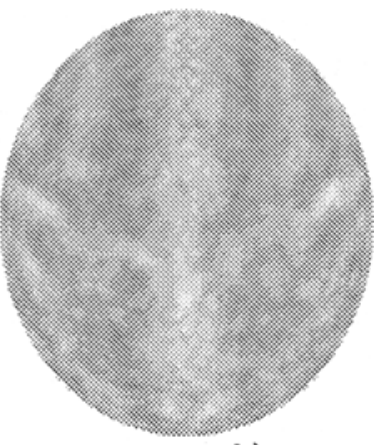

b)

Fig. 4. a) Right view, and b) Top View of thickness of cortex from Fig. 2a mapped onto an ellipsoid,(range: $3 \mathrm{~mm}$ to $7 \mathrm{~mm}$ ).

\subsection{Cortical Thickness Map}

The thickness of the gray matter can be measured at any point on an individual cortical surface, using the two deformed surfaces, and averaged over the entire set of surfaces, shown on an ellipsoid in Fig. 4. However, the resulting thickness is biased towards the original proximity constraints defined in the dual-surface model. In order to minimize this bias, the current estimate of local cortical thickness should be used to define a second-generation model and the surface deformation process repeated for all 151 surfaces. Such refinement of the cortical thickness map may be repeated until a convergence is achieved. More work is required to determine to what extent the $a$ priori constraints and the deformation algorithm itself control the perceived thickness of the cortex.

\section{$5 \quad$ Summary}

A novel method of surface deformation for image segmentation and matching has been presented, with the following features:

- a boundary search along the local surface normal is used to increase the range of attraction of edges,

- the use of proximity constraints with appropriate weights guarantees avoidance of intersecting surface geometries,

- proximity constraints provide an alternative to the conventional method of regularization by stretching and bending constraints,

- intuitive geometric constraints are used in place of arbitrary weights,

- multiple surfaces, models, and datasets may be combined into a single objective function,

- and finally, the application of this surface deformation method to the identification of the total cerebral cortical surface from MR images is achieved with improved insensitivity to partial volume effects. 
The ability to automatically generate surface represenations from images provides opportunities for sophisticated analysis of large populations of neuroanatomical data. Generation of average surface models which incorporate descriptions of mean position, shape, and thickness is in progress. Future directions include improving the mapping of cortical surface between subjects, as well as sulcal recognition, and application to identification of cerebellum, brainstem, and other neuroanatomic structures.

\section{References}

1. M. Kass, A. Witkin, and D. Terzopoulos, "Snakes: Active contour models," International Journal of Computer Vision, pp. 321-331, 1988.

2. D. Collins, T. Peters, W. Dai, and A. Evans, "Model-based segmentation of individual brain structures from mri data," in Proceedings Visualization in Biomedical Computing 1992, pp. 10-23, 1992.

3. C. Davatzikos and R. N. Bryan, "Using a deformable surface model to obtain a shape representation of the cortex," Proceedings of the IEEE International Conference on Computer Vision 1995, pp. 212-217, 1995.

4. S. Sandor and R. Leahy, "Surface-based labeling of cortical anatomy using a deformable atlas," IEEE Transactions on Medical Imaging, vol. 16, pp. 41-54, Feb 1997.

5. L. D. Cohen and I. Cohen, "Finite-element methods for active contour models and balloons for 2-d and 3-d images," IEEE Transactions on Pattern Analysis and Machine Intelligence, vol. 15, pp. 1131-1147, Nov 1993.

6. L. Staib and J. Duncan, "Deformable fourier models for surface finding in 3d images," in Proceedings Visualization in Biomedical Computing 1992, pp. 90-104, 1992.

7. T. McInerney and D. Terzopoulos, "Deformable models in medical image analysis: a survey," Medical Image Analysis, vol. 1, no. 2, 1996.

8. M. Vaillant, C. Davatzikos, and N. R. Bryan, "Finding 3d parametric representations of the deep cortical folds," Proceedings of the Workship on Mathematical Methods in Biomedical Image Analysis 1996, pp. 151-159.

9. A. M. Dale and M. I. Sereno, "Improved localization of cortical activity by combining eeg and meg with mri cortical surface reconstruction: a linear approach," Journal of Cognitive Neuroscience, vol. 5, pp. 162-176, 1993.

10. J. G. Sled, A. P. Zijdenbos, and A. C. Evans, "A comparison of retrospective intensity non-uniformity correction methods for MRI," in Information Processing in Medical Imaging, 1997. in press.

11. D. Collins, P. Neelin, T. Peters, and A. Evans, "Automatic 3d intersubject registration of $\mathrm{mr}$ volumetric data in standardized talairach space," Journal of Computer Assisted Tomography, vol. 18, pp. 192-205, Mar. 1994.

12. R. Bajcsy and K. cič, "Multiresolution elastic matching," Computer Vision, Graphics, and Image Processing, vol. 46, pp. 1-21, 1989.

13. C. Nastar and N. Ayache, "Non-rigid motion analysis in medical images: a physically based approach," in Proceedings of Information Processing in Medical Imaging, (Flagstaff, Arizona), pp. 17-32, June 1993. 